Publ. RIMS, Kyoto Univ.

40 (2004), 731-756

\title{
Temperature as Order Parameter of Broken Scale Invariance ${ }^{\dagger}$
}

By

\author{
Izumi OJIMA*
}

\begin{abstract}
In algebraic quantum field theory the (inverse) temperature is shown to be a macroscopic order parameter to parametrize mutually disjoint thermal sectors arising from the broken scale invariance under renormalization-group transformations.

This is accomplished in a mathematical formalism for the consistent treatment of explicitly broken symmetries such as broken scale invariance, on the basis of a clearcut criterion for the symmetry breakdown in a unified scheme for sectors proposed recently by the author.
\end{abstract}

\section{§1. Introduction}

The purpose of the present paper is to give the explanation and the proof of the following statement:

Theorem 1.1. In the standard setting up of algebraic quantum field theory, the inverse temperature $\beta:=\left(\beta^{\mu} \beta_{\mu}\right)^{1 / 2}$ is a macroscopic order $\boldsymbol{p a}$ rameter for parametrizing mutually disjoint sectors in the thermal situation arising from the broken scale invariance under the renormalization-group transformations, where $\beta^{\mu}$ is an inverse temperature 4-vector of a relativistic KMS state $\omega_{\beta^{\mu}}$ describing a thermal equilibrium in its rest frame.

This is obtained in my recent attempts to facilitate smooth accesses to the mathematical methods of algebraic quantum field theory (QFT for short) for their use in the actual problems in physics in such forms as

Communicated by T. Kawai, November 4, 2003. Revised December 11, 2003.

2000 Mathematics Subject Classification(s): 81R40, 81T05, 81T17, 82B10, 82B28

Supported by JSPS Grants No. 15540117

${ }^{\dagger}$ This article is an invited contribution to a special issue of Publications of RIMS commemorating the fortieth anniversary of the founding of the Research Institute for Mathematical Sciences.

* Research Institute for Mathematical Sciences, Kyoto University, Kyoto 606-8502, Japan. 
a) a mathematical formulation of explicitly broken symmetries such as the above mentioned broken scale invariance, and,

b) an attempt for the mathematical understanding of mutual relations among quantum, thermal and geometrical aspects embodied in QFT in various physical contexts involving such key notions as renormalization group and order parameters, etc.

In the following the above two results crucial for the explanation of the main theorem are shown to be naturally understood in a unified scheme proposed in my recent projects for controlling the mutual relations between the micro- and macroscopic aspects involved in quantum physics from the viewpoint of superselection structures. For this purpose, first let me start with a brief survey of the essence of the results obtained in [20], in combination with some standard basic materials and tools available in the algebraic QFT necessary for our purpose.

The proposed scheme in [20] is meant for treating general sector structures on the basis of selection criteria. It has been extracted from a general formulation of non-equilibrium local states [5] and adapted to the discussions of the sector structures arising from internal symmetries without and with their spontaneous breakdown. In the original Doplicher-Roberts (DR) theory [9], the global gauge group $G$ of an internal symmetry and the field algebra $\mathfrak{F}$ consisting of $G$-non-singlet quantum fields $\hat{\varphi}^{i}(x)$ are shown to be recovered from the algebra $\mathfrak{A}=\mathfrak{F}^{G}$ of $G$-singlet observables in combination with the data on states of physical relevance on $\mathfrak{A}$ selected by the Doplicher-Haag-Roberts (DHR) criterion [8] as states with localizable charges. Previously, this theory was satisfactorily formulated only in the cases with unbroken symmetries, and its general significance was not properly understood owing to its mathematical sophistication. In [20], such general essence extracted from this theory has been extended not only to the broken symmetries but also to such thermal situations as involving non-equilibrium local states in QFT [5]; through these results a unified theoretical scheme to control micro-macro transitions is seen to emerge, on the basis of categorical adjunctions as matching conditions between selected states and reference states for comparison which are realized by 'solving' the selection criteria. By these attempts, it has turned out that the proposed scheme allows one to treat various different physical theories specialized to each specific domain in nature, in quite a homogeneous and unified way, such as QFT in the vacuum situation, statistical mechanics of quantum fields at finite temperatures and their non-equilibrium states with non-trivial space-time dependent structures and behaviours in the following forms: 
1. DHR-DR superselection theory [8,9] and its reformulation [20],

2. extension to the situations with a spontaneously broken internal symmetry $[20]$,

3. general formulation of non-equilibrium local states in QFT $[5,18,19]$.

According to a suitable choice of a selection criterion, we select all the physical states of relevance to each physical domain or aspects of interest to be described theoretically, from the set of all generic states on the net of quantum local observables or the algebra of quantum fields. The meaningful choices of the selection criteria have turned out (see [20]) to involve some standard reference systems, such as the group dual $\hat{G}$ in the above case of DHR-DR superselection theory controlling the mutual relations between the observable algebra $\mathfrak{A}=\mathfrak{F}^{G}$ with a specified family $\mathcal{T}$ of physical states with localizable charges and the non-commutative dynamical system $\mathfrak{F} \curvearrowleft G$ with the group of internal symmetry $G=\operatorname{Gal}(\mathfrak{F} / \mathfrak{A})$ given as a Galois group. In the case of nonequilibrium local states, such a role is played by the space $B_{K}$ of thermodynamic parameters $(\beta, \mu)$ and that $M_{+}\left(B_{K}\right)$ of probability measures $\rho$ on $B_{K}$ describing the fluctuations of $(\beta, \mu)$. Through the comparison of a generic unknown state $\omega$ with members of standard states $\omega_{\rho}=\mathcal{C}^{*}(\rho)$ equipped with parameters $\rho$ belonging to the reference system, we can judge whether $\omega$ satisfies the criterion or not. If the selection criterion and the standard reference system are suitably set up, we can solve an "inverse" of a map $\mathcal{C}^{*}(c \rightarrow q$ channel) from the reference system to the set of generic quantum states as a kind of "left adjoint" $(q \rightarrow c$ channel) in the categorical adjunction which provides us with the interpretation of a generic selected state $\omega$ in terms of the vocabulary of the standard known object $\rho$ belonging to the reference system. Here what plays crucial roles is the relation (Sec.3) between the superselection sectors arising from the classification of states and representations of the algebra of quantum physical variables and the spectrum of the centre (of the universal representation containing all the selected relevant ones), the latter of which plays the roles of macroscopic order parameters to distinguish among different sectors on the basis of their mutual disjointness.

If this kind of machinery works appropriately, then it will allow us to analyze in terms of the selection criteria the mutual relations among different theories to describe different physical domains or aspects, on the basis of which we can attain a framework to allow one specific form of a theory adapted to a fixed scale region in the physical world to be freely transferred to another one, according to the changes in length scales and aspects relevant to the problems in 
question. At this point, however, we note that all the above results are obtained in the essential use of the basic notions and mathematical techniques developed on the notions of vacuum states (characterized by the spectrum condition) and/or KMS states (based on the KMS condition), which have been adopted as they stand without any re-considerations. Namely, the most important case of the family of KMS states (including the vacuum states as a special case of $\beta=\infty$ or $T=0$ ) as standard reference systems has been untouched, left outside the context of the above proposed scheme, without a physically natural reformulation. It is clear that we cannot attain the aim of a unified scheme in a self-consistent way unless this case is successfully incorporated in the framework briefly explained above.

For this purpose, we start to approach to this question by examining the geometric and algebraic meanings of the temperatures, asking ourselves a question, "Is a temperature a priori parameter or physical quantity?". As will be examined in the next section, the most useful hint can be found in the famous Takesaki theorem [25] showing the mutual disjointness $\omega_{\beta_{1}} \circ \omega_{\beta_{2}}$ between KMS states at different temperatures, $\beta_{1} \neq \beta_{2}$, for a quantum $\mathrm{C}^{*}$-dynamical system with type III representations in its KMS states.

The general and physical meanings contained in the mathematical notions of disjointness will be explained in Sec.3 in combination with the related important and basic notions such as quasi-equivalence of representations, folia and the role of the spectrum of the centre in classifying mutually disjoint representations and states. On the basis of these preparations, the criterion for symmetry breakdown is presented in Sec.4. To adapt the above ingredients to the situation with the broken scale invariance in thermal situations, an augmented algebra as a composite system of a genuine quantum system and a classical macroscopic system is constructed in Sec.5 to accommodate spontaneously and/or explicitly broken symmetries unifying the viewpoint presented in Sec. 4 and the notion of scaling nets and algebras due to Buchholz and Verch [6]. In use of the mappings to relate states on the original quantum system and the augmented one, the claimed result on the role of (inverse) temperature as an order parameter of broken scale invariance is proved in Sec.6. The final section summarizes these consequences and briefly mentions the related interesting problems in this context to be further investigated.

\section{§2. Temperature: A Priori Parameter or Physical Quantity?}

When an object in thermal equilibrium with respect to its rest frame is moving relative to our frame, we observe in it such non-equilibrium features 
as heat current just for the kinematical reason. This means that the thermal equilibrium is meaningful only in reference to the frame in which the object is at rest, and it can be shown [16] to imply the spontaneous breakdown of Lorentz boost symmetry in the context of (special-)relativistic QFT. In this situation, a Lorentz 4-vector $\beta^{\mu}$ of inverse-temperature is shown to be a key member of necessary parameters for specifying a thermal equilibrium state:

$$
\beta^{\mu}=\beta u^{\mu} \in V_{+}, \quad \beta:=\left(\beta^{\mu} \beta_{\mu}\right)^{1 / 2}=\left(k_{B} T\right)^{-1},
$$

where $V_{+}$denotes the (open) forward lightcone in the Minkowski space defined by $V_{+}:=\left\{\left(x^{\mu}\right) \in \mathbb{R}^{4} ; x \cdot x=x^{0} x^{0}-\vec{x} \cdot \vec{x}>0, x^{0}>0\right\}$, and $k_{B}$ and $T$ are the Boltzmann constant and a temperature, respectively. Such a thermal state $\omega_{\left(\beta^{\mu}\right)}$ parametrized by $\beta^{\mu}$ is shown [3] to be characterized by a relativistic KMS condition, a relativistic extension of the standard KMS condition [2], and will be called a relativistic KMS state or simply a KMS state hereafter.

From the above explanation, the time-like unit vector $u^{\mu}:=\beta^{\mu} / \beta\left(u^{\mu} u_{\mu}=\right.$ 1 ) is seen to describe a relative velocity for specifying the rest frame in which the relativistic KMS state $\omega_{\left(\beta^{\mu}\right)}$ exhibits its genuine thermal equilibrium nature, and it represents an order parameter associated with this spontaneous symmetry breaking (SSB) of Lorentz boosts [16]. In the context of non-equilibrium local states [5], a non-trivial spacetime-dependent temperature distribution $x \longmapsto \beta^{\mu}(x) \in V_{+}$is allowed to appear, in which $x \longmapsto u^{\mu}(x)=\beta^{\mu}(x) / \beta(x)$ becomes a time-like member of the vierbein field to specify the rest frame at each spacetime point $x$. Putting this geometric aspect in a more general context of QFT formulated in a curved spacetime (i.e., non-equilibrium states in a curved background spacetime $[19,17])$, we will encounter interesting mathematicalphysical problems at the boundary of geometry and thermodynamics.

The problem to be discussed here is, however, a question concerning another factor $\beta=\left(\beta^{\mu} \beta_{\mu}\right)^{1 / 2}$ in the formula $\beta^{\mu}=\beta u^{\mu} \in V_{+}$, the inverse temperature itself as a Lorentz scalar: is $\beta$ any kind of order parameter related with a certain symmetry similarly to the case of $u^{\mu}$ ? What is suggestive in this context is the following famous theorem due to Takesaki [25] (see, for instance $[2])$ :

Theorem 2.1 (Takesaki). Let $\left(\mathfrak{A}_{\alpha}^{\curvearrowleft} \mathbb{R}\right)$ be a $C^{*}$-dynamical system, and suppose that $\omega_{1}$ and $\omega_{2}$ are KMS-states corresponding to two different values $\beta_{1}, \beta_{2} \in \mathbb{R}$. Assume that $\pi_{\omega_{1}}(\mathfrak{A})^{\prime \prime}$ is a type-III von Neumann algebra. It follows that the states $\omega_{1}$ and $\omega_{2}$ are disjoint.

This mathematical fact suggests the following physical picture for such quantum systems as QFT with infinite degrees of freedom, which intrinsically 
involve type-III von Neumann algebras (as representation-independent local subalgebras [11] and/or in thermal situations [14]): according to standard results $[7,24]$ in the representation theory of $\mathrm{C}^{*}$-algebras, a family of disjoint representations generate a non-trivial centre in the representation containing them as subrepresentations, whose elements can be regarded as macroscopic order parameters because of their mutual commutativity and of their role in discriminating among different representations within the family according to its spectrum. Since all the GNS representations of the KMS states of QFT except for $\beta=0, \infty$ are known to be type-III von Neumann algebras, we are naturally led to a situation with continuous superselection sectors formed by KMS states at different temperatures, distinguished mutually by macroscopic central observables (in a representation containing all the KMS states) among which the (inverse) temperature $\beta$ is found. Namely, $\beta$ becomes in this situation a physical macro-variable running over the space of all possible thermal equilibria, instead of being an a priori given fixed parameter as is treated in the standard approach to statistical mechanics.

Starting from this observation, it will be shown in the following that $\beta$ is a physical order parameter corresponding to the spontaneously or explicitly broken scale invariance under the renormalization-group transformations; namely, $\beta$ 's not only parametrize continuous sectors of thermal equilibria at different temperatures, but also are mutually interrelated by the renormalization-group transformation associated with the broken scale invariance, which clarifies the geometric structure of the thermodynamic classifying space identified with the spectrum of the above centre of the representation universal within the KMS family.

\section{§3. Classification of Representations and States: Central Spectrum as Classifying Space of Sectors}

Just for convenience' sake, let us briefly recall and summarize the basic mathematical notions relevant to the present context in the following form.

1) Folium/disjointness/quasi-equivalence:

Let $\mathfrak{A}$ be a unital $\mathrm{C}^{*}$-algebra and denote by $E_{\mathfrak{A}}$ the set of all states on $\mathfrak{A}$ defined as normalized positive linear functionals. All representations $(\pi, \mathfrak{H})$ are to be understood here as unital *-representations in the sense that $\pi(\mathbf{1})=\mathbf{1}_{\mathfrak{H}}$, $\pi\left(A^{*}\right)=\pi(A)^{*}$.

Definition 3.1 (Folium). Given a representation $(\pi, \mathfrak{H})$ of $\mathfrak{A}$, a state $\varphi \in E_{\mathfrak{A}}$ is called $\pi$-normal if there exists a density operator in $\mathfrak{H}$ s.t. $\varphi(A)=$ 
$\operatorname{Tr}(\sigma \pi(A))(\forall A \in \mathfrak{A})$. Totality $\mathfrak{f}(\pi)$ of $\pi$-normal states is called a folium of $\pi$ :

$$
\mathfrak{f}(\pi):=\{\mathfrak{A} \ni A \longmapsto \operatorname{Tr}(\sigma \pi(A)) ; \sigma \text { : density operator in } \mathfrak{H}\} .
$$

A folium $\mathfrak{f}(\omega)$ of a state $\omega \in E_{\mathfrak{A}}$ is defined by $\mathfrak{f}(\omega):=\mathfrak{f}\left(\pi_{\omega}\right)$ w.r.t. the GNS representation $\pi_{\omega}$ of $\omega$.

From the definition, it is clear that the linear span of a folium gives the predual $\left(\pi(\mathfrak{A})^{\prime \prime}\right)_{*}$ of the von Neumann algebra $\pi(\mathfrak{A})^{\prime \prime}$, consisting of $\sigma$-weakly continuous linear functionals on $\pi(\mathfrak{A})^{\prime \prime}: \operatorname{Lin}(\mathfrak{f}(\pi))=\left(\pi(\mathfrak{A})^{\prime \prime}\right)_{*}, \operatorname{Lin}(\mathfrak{f}(\pi))^{*}=$ $\pi(\mathfrak{A})^{\prime \prime}$. In terms of this notion, the definitions of disjointness and quasiequivalence of representations can be understood in a clear-cut way, as follows.

Definition 3.2 (Disjointness [7]). Two representations $\left(\pi_{1}, \mathfrak{H}_{1}\right),\left(\pi_{2}, \mathfrak{H}_{2}\right)$ of $\mathfrak{A}$ are said to be disjoint and written as $\pi_{1} \circ \pi_{2}$, if and only if they have no pair $\rho_{1}, \rho_{2}$ of unitarily equivalent non-trivial subrepresentations $0 \neq \rho_{1} \prec$ $\pi_{1}, 0 \neq \rho_{2} \prec \pi_{2}$, Likewise, states $\omega_{1}, \omega_{2} \in E_{\mathfrak{A}}$ with disjoint GNS representations $\pi_{\omega_{1}} \circ \pi_{\omega_{2}}$ are said to be disjoint and written as $\omega_{1} \circ \omega_{2}$.

According to the standard results (see [7]), the defined disjointness is rephrased into the following equivalent forms:

$$
\begin{aligned}
\pi_{1} \circ \pi_{2} & \Longleftrightarrow \mathfrak{f}\left(\pi_{1}\right) \cap \mathfrak{f}\left(\pi_{2}\right)=\emptyset \\
& \Longleftrightarrow \mathcal{H}^{\mathfrak{A}}\left(\pi_{2} \leftarrow \pi_{1}\right)=0 \\
& \Longleftrightarrow c\left(P\left(\pi_{1}\right)\right) \perp c\left(P\left(\pi_{2}\right)\right),
\end{aligned}
$$

where $\mathcal{H}^{\mathfrak{A}}\left(\pi_{2} \leftarrow \pi_{1}\right)$ is the set of intertwiners from $\pi_{1}$ to $\pi_{2}$ defined by

$$
\mathcal{H}^{\mathfrak{A}}\left(\pi_{2} \leftarrow \pi_{1}\right):=\left\{T \in B\left(\mathfrak{H}_{1}, \mathfrak{H}_{2}\right) ; T \pi_{1}(A)=\pi_{2}(A) T(\forall A \in \mathfrak{A})\right\}
$$

and $P\left(\pi_{i}\right) \in \pi(\mathfrak{A})^{\prime}=\mathcal{H}^{\mathfrak{A}}(\pi \leftarrow \pi)(i=1,2)$ are projections corresponding to $\pi_{i}$ regarded as subrepresentations of a common representation $\pi$ (e.g., $\pi=$ $\left.\pi_{1} \oplus \pi_{2}\right)$ and $c(P)$ denotes the central support $c(P):=\min \{F$ : projection $\left.\in \pi(\mathfrak{A})^{\prime \prime} \cap \pi(\mathfrak{A})^{\prime} ; F \geq P\right\}$ of a projection $P\left(\in \pi(\mathfrak{A})^{\prime}\right.$ or $\left.\pi(\mathfrak{A})^{\prime \prime}\right)$. If $\pi_{1}, \pi_{2}$ are both irreducible, disjointness $\pi_{1} \circ \pi_{2}$ means simply their unitary inequivalence. If both $\pi_{1}, \pi_{2}$ can be uniquely decomposed into irreducible components, $\pi_{1}, \pi_{2}$ have no common irreducible components.

The 'opposite' situation to the disjointness is given by the notion of quasiequivalence defined next on the basis of the following proposition:

Proposition 3.1 ([7]). $\quad$ The following conditions for representations $\pi_{1}$, $\pi_{2}$ of $\mathfrak{A}$ are all equivalent: 
(i) No non-trivial subrepresentation of $\pi_{1}$ is disjoint from $\pi_{2}$ and no non-trivial subrepresentation of $\pi_{2}$ is disjoint from $\pi_{1}$ [negation of disjointness];

(ii) $\exists \Phi: \pi_{1}(\mathfrak{A})^{\prime \prime} \rightarrow \pi_{2}(\mathfrak{A})^{\prime \prime}:$ isomorphism of von Neumann algebras s.t. $\pi_{2}(A)=\Phi\left(\pi_{1}(A)\right)$ for $\forall A \in \mathfrak{A} ;$

(iii) $\exists n_{1}, n_{2}$ : cardinals s.t. $n_{1} \pi_{1} \cong n_{2} \pi_{2}$ where $n_{1} \pi_{1}$ and $n_{2} \pi_{2}$ are, respectively, multiples of $\pi_{1}$ and of $\pi_{2}$ [i.e., unitary equivalence up to multiplicities];

(iv) $\mathfrak{f}\left(\pi_{1}\right)=\mathfrak{f}\left(\pi_{2}\right)$ for the folia of $\pi_{1}, \pi_{2}$.

If $\pi_{1}, \pi_{2}$ are subrepresentations of a representation $\pi$ with the corresponding projections $P\left(\pi_{1}\right), P\left(\pi_{2}\right) \in \pi(\mathfrak{A})^{\prime}$, the above (i)-(iv) are also equivalent to the next $(\mathrm{v})$ :

(v) $c\left(P\left(\pi_{1}\right)\right)=c\left(P\left(\pi_{2}\right)\right)$ for the central supports of $P\left(\pi_{1}\right), P\left(\pi_{2}\right)$.

Definition 3.3 (Quasi-equivalence). Two representations $\pi_{1}, \pi_{2}$ satisfying one (and hence, all) of the conditions (i)-(v) are said to be quasiequivalent, and written as $\pi_{1} \approx \pi_{2}$. States $\omega_{1}$ and $\omega_{2}$ are said to be quasiequivalent if the corresponding GNS representations $\pi_{\omega_{1}}$ and $\pi_{\omega_{2}}$ are quasiequivalent.

2) Pure phase vs. mixed phase; superselection sectors and order parameter

While KMS states $\omega_{\beta}$ describing thermal equilibria are all mixed states (except for the case of vacuum with $\beta=\infty$ ), their decompositions into pure states are highly non-unique for quantum dynamical systems with infinte degrees of freedom because of their type-III-ness. Therefore, it is more legitimate to understand a KMS state as an entity in itself without reference to pure states. For this purpose, we need to know the minimal units of KMS states in order for a generic one to be decomposed canonically. Such a unit is known to be found in a thermodynamic pure phase $\omega \in E_{\mathfrak{A}}$ characterized by ergodicity, or equivalently by factoriality defined by the triviality of centre $\mathfrak{Z}_{\omega}(\mathfrak{A})=\pi_{\omega}(\mathfrak{A})^{\prime \prime} \cap \pi_{\omega}(\mathfrak{A})^{\prime}=\mathbb{C I}_{\mathfrak{H}_{\omega}}$ as a condition equivalent to the extremality in the set $K_{\beta}$ of all KMS states at $\beta$ on $\mathfrak{A} \curvearrowleft \mathbb{R}[2]$.

Along this line, we call pure phases any factor states $\omega$ or factor representations with trivial centre. If a given state $\omega$ is not a pure phase, it is called a mixed phase whose GNS representation $\pi_{\omega}$ has a non-trivial centre $\mathfrak{Z}_{\omega}(\mathfrak{A})$. As a commutative algebra, the centre $\mathfrak{Z}_{\omega}(\mathfrak{A})$ admits a "simultaneous diagonalization" due to the well-known Gel'fand theorem expressing it as a 
function algebra $L^{\infty}\left(\operatorname{Spec}\left(\mathfrak{Z}_{\omega}(\mathfrak{A})\right)\right)$ on the spectrum $\operatorname{Spec}\left(\mathfrak{Z}_{\omega}(\mathfrak{A})\right)$ consisting of characters or maximal ideals. Corresponding to this, $\omega$ and $\pi_{\omega}$ are canonically decomposed (= central decomposition) into factor states (= pure phases) and factor subrepresentations (= sectors):

$$
\begin{aligned}
\omega(A) & =\int_{S p\left(\mathfrak{Z}_{\omega}(\mathfrak{A})\right)} \omega_{\lambda}(A) d \mu(\lambda), \\
\pi_{\omega}(A) & =\int_{S p\left(\mathfrak{Z}_{\omega}(\mathfrak{A})\right)}^{\oplus} \pi_{\omega_{\lambda}}(A) d \mu(\lambda) .
\end{aligned}
$$

According to the above proposition, any pair of factor states or factor representations are either quasi-equivalent or disjoint and if pure phases $\omega_{1}, \omega_{2}$ are disjoint, there exists $\exists C \in \mathfrak{Z}_{\pi}(\mathfrak{A})$ s.t. $\omega_{1}(C) \neq \omega_{2}(C)$ within a representation $\pi$ containing $\pi_{\omega_{1}} \oplus \pi_{\omega_{2}}$. In this sense, each point of $\operatorname{Spec}\left(\mathfrak{Z}_{\omega}(\mathfrak{A})\right)$ represents a realized value of parameters to distinguish among different pure phases contained in a mixed phase $\omega$, and hence, each central element $C \in \mathfrak{Z}_{\omega}(\mathfrak{A})$ can be identified with a macroscopic order parameter.

Therefore, a mixed phase represents just the situation of a superselection rule consisting of sectors each of which is identified with a folium of pure phases or its factor representations labelled by a point in $\operatorname{Spec}\left(\mathfrak{Z}_{\omega}(\mathfrak{A})\right)$.

3) Scheme for classifying microscopic sectors by means of macroscopic order parameters

The above observations naturally lead to a "unified scheme for generalized sectors based upon selection criteria" [19] which can be schematized as follows:

i) $\left[\left(q: \begin{array}{c}\text { generic objects } \\ \text { to be selected }\end{array}\right] \Longrightarrow\right.$ ii) $\left[\begin{array}{c}\text { standard reference system with } \\ \text { classifying space of sectors }\end{array}(: c)\right]$ iii) comparison of i) with ii)

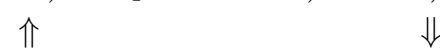

iv) $\left[\begin{array}{c}\text { selection criterion: } \\ \text { ii) } \underset{c-q \text { channel }}{\Longrightarrow} \text { i) }\end{array}\right] \underset{\text { adjunction }}{\stackrel{\text { categorical }}{\rightleftarrows}}\left[\begin{array}{c}\text { interpretation of i) in } \\ \text { terms of ii): i) } \underset{q-c \text { channel }}{\stackrel{\Longrightarrow}{\rightleftharpoons}} \text { ii) }\end{array}\right]$

The applicability of this scheme has been confirmed in [20] in three major situations, the DHR superselection rule $[8,9]$ to explain the operational origin of internal symmetries, its extension to SSB cases [20] and the formulation of non-equilibrium local states in QFT $[5,18,19]$. In all three cases, the item 
i) is given by the set of states on the net $\mathcal{O} \longmapsto \mathfrak{A}(\mathcal{O})$ of local observables or its global algebra $\mathfrak{A}$ to be explained in the next section (or, a version of it slightly restricted w.r.t. the energy spectrum in the case of non-equilibrium local states $[19,20])$. The item ii) is chosen in the discussion of non-equilibrium local states as the set $K:=\operatorname{Conv}\left(\cup_{\beta \in V_{+}} K_{\beta}\right)$ of all the convex combinations of (relativistic) KMS states $\in K_{\beta}$ at all possible inverse temperatures $\beta \in V_{+}$. In contrast, the corresponding choices in the DHR-DR theory and its extension to SSB are not known a priori, which turn out through the analyses to be, respectively, the group dual $\hat{G}$ (or functions $l^{\infty}(\hat{G})$ on it) of the group $G$ of the arising internal symmetry and the sections $l^{\infty}\left(\amalg_{g H \in G / H} g \hat{H} g^{-1}\right)$ of a fibre bundle related with the group $H$ of unbroken remaining symmetry and that $G$ of the spontaneously broken symmetry. The item iii) is just to compare a given unknown generic state $\omega$ in i) with those special states in i) sent from the standard reference system ii) by the embedding map as a c-q channel ii) $\Longrightarrow$ i). If $\omega$ is judged to be identified with one of such embedded reference states, then the 'inverse' map as a q-c channel i) $\Longrightarrow$ ii) provides the interpretation of $\omega$ in terms of ii). In this way, the $G$-charge contents of $\omega$ in the DHR-DR case is described in terms of the (fluctuation probability over) unitary equivalence classes $\subset \hat{G}$, and, in the SSB case, in terms of the data of $g H \in G / H$ specifying the position (within the family of degenerate vacua parametrized by $G / H$ ) of a vacuum sector (e.g., the spatial direction of the magnetization in the example of a Heisenberg ferromagnet) to which $\omega$ belongs, in combination with the $H$-charge contents of $\omega$. In the case of non-equilibrium local states, $\omega$ is characterized as such a state if it shows the agreement for certain restricted class of pointlike quantum thermal observables (defined by some asymptotic limits of elements in the local net $\mathcal{O} \longmapsto \mathfrak{A}(\mathcal{O})$ converging to a spacetime point $\left.x \in \mathbb{R}^{4}\right)$ with a standard state of such a form as $\omega_{\rho}=\int_{B_{K}} d \rho(\beta, \mu) \omega_{\beta, \mu}$ with $\rho \in M_{+}\left(B_{K}\right)$ describing the statistical fluctuations of thermodynamic parameters $(\beta, \mu) \in B_{K}$ over various thermodynamic pure phases. In this case, $d \rho(\beta, \mu)$ gives the thermal interpretation of a generic non-equilibrium state $\omega$ at a point $x$.

Since all these are in use of some systematic techniques available for vacuum states and/or KMS states based upon their mathematical formulation, it is quite important to try the possibility to treat all the KMS states (including $\beta=\infty$ as a vacuum) themselves just according to the same spirit as above. 


\section{$\S 4$. Criterion for Symmetry Breakdown}

To show that the inverse temperature appears as an order parameter of broken scale invariance, we need to give precise formulations of

a) the scale transformations which may possibly be allowed to involve explicit breaking effects such as the presence of non-vanishing mass terms,

and of

b) a criterion for symmetry breakdown,

and then to exhibit

c) the role of the inverse temperature as an order parameter for this broken scale invariance.

For this purpose, we start here with the criterion for a spontaneous symmetry breaking (SSB), as a special case of b) applicable to the symmetries described by a strongly continuous automorphic action $\tau$ of a locally compact group $G$ on the $\mathrm{C}^{*}$-algebra $\mathfrak{F}$ of quantum fields: $G \underset{\tau}{\curvearrowright} \mathfrak{F}$. To be more precise, the algebraic formulation of QFT is usually not based upon a $\mathrm{C}^{*}$-algebra $\mathfrak{F}$ of quantum fields which are in general not directly observable owing to their non-trivial behaviours under the group $G$ of an internal symmetry. Instead, the basic ingredient to play the principal roles is a net $\mathcal{O} \longmapsto \mathfrak{A}(\mathcal{O})$ of local observables with each local subalgebra $\mathfrak{A}(\mathcal{O})$ defined as a $\mathrm{W}^{*}$-algebra; any self-adjoint element $A=A^{*} \in \mathfrak{A}(\mathcal{O})$ is supposed to correspond to a physical quantity, experimentally observable within a spacetime region $\mathcal{O}$ chosen from a suitable family of spacetime regions which constitute a directed set w.r.t. the inclusion relation, the typical choice being the family $\mathcal{K}:=\left\{\left(b+V_{+}\right) \cap\left(c-V_{+}\right) ; a, b \in \mathbb{R}^{4}\right\}$ of double cones in the Minkowski spacetime $\mathbb{R}^{4}$. The common properties to be naturally satisfied by this net are taken as follows (see, for instance, [12]):

i) "Isotony": for $\mathcal{O}_{1}, \mathcal{O}_{2} \in \mathcal{K}$, the inclusion relation $\mathcal{O}_{1} \subset \mathcal{O}_{2}$ should imply $\mathfrak{A}\left(\mathcal{O}_{1}\right) \subset \mathfrak{A}\left(\mathcal{O}_{2}\right)$, on the basis of which the global algebra $\mathfrak{A}=\frac{\mathcal{K}_{\mathcal{O}} / \mathbb{R}^{4}}{\mathfrak{A}(\mathcal{O})}{ }^{\|\cdot\|}$ of observables can be defined as the $\mathrm{C}^{*}$-inductive limit of all the local subalgebras $\mathfrak{A}(\mathcal{O}), \mathcal{O} \in \mathcal{K}$.

ii) Relativistic covariance: the family $\mathcal{K}$ can be regarded as a category consisting of objects as double cones $\mathcal{O} \in \mathcal{K}$ and of arrows $\mathcal{O} \stackrel{(a, \Lambda)}{\longrightarrow}(a, \Lambda) \mathcal{O}$ defined by the elements $(a, \Lambda) \in \mathcal{P}_{+}^{\uparrow}=\mathbb{R}^{4} \rtimes L_{+}^{\uparrow}$ of the Poincaré group acting on the Minkowski spacetime $\mathbb{R}^{4}$ and $\mathcal{K}$, respectively, by $[(a, \Lambda) x]^{\mu}=\Lambda_{\nu}^{\mu} x^{\nu}+a^{\mu}$, 
$(a, \Lambda) \mathcal{O}=\Lambda \mathcal{O}+a$. Then, the local net $\mathcal{O} \longmapsto \mathfrak{A}(\mathcal{O})$ should constitute a functor $\mathfrak{A}: \mathcal{K} \rightarrow A l g[17,4]$ from the category $\mathcal{K}$ to the category $A l g$ of $\mathrm{W}^{*}$-algebras whose arrows are (normal) *-homomorphisms:

$$
\begin{gathered}
\mathcal{K} \ni \mathcal{O} \rightarrow \mathfrak{A}(\mathcal{O}) \in A l g \\
(a, \Lambda) \downarrow \circlearrowleft \quad \downarrow \alpha_{(a, \Lambda)}:=\mathfrak{A}(a, \Lambda) \\
\Lambda \mathcal{O}+a \rightarrow \mathfrak{A}(\Lambda \mathcal{O}+a)
\end{gathered}
$$

iii) Local commutativity (as a mathematical formulation of Einstein causality): for spacelike separated regions $\mathcal{O}_{1}, \mathcal{O}_{2} \in \mathcal{K}$ (i.e., $(x-y) \cdot(x-y)<0$ $\left.\left(\forall x \in \mathcal{O}_{1}, \forall y \in \mathcal{O}_{2}\right)\right)$ the local subalgebras $\mathfrak{A}\left(\mathcal{O}_{1}\right)$ and $\mathfrak{A}\left(\mathcal{O}_{2}\right)$ are commuting in the sense of $A B=B A\left(\forall A \in \mathfrak{A}\left(\mathcal{O}_{1}\right), \forall B \in \mathfrak{A}\left(\mathcal{O}_{2}\right)\right)$.

The basic standpoint of the algebraic QFT (though not completely implemented yet) is to regard the algebra $\mathfrak{F}$ of unobservable quantum fields acted upon by a group $G$ of internal symmetry as a kind of mathematical device constructed by the method of Galois extension from $\mathfrak{A}$ by 'solving some equations' identified with a suitably chosen criterion to select out a family of physically relevant states $[8,9]$. This strategy has definite merits in providing a clear picture for the mutual relations between the two aspects involving spacetime(=external) and internal symmetries, treating the former in such a form as the spacetime dependence of the net $\mathcal{O} \longmapsto \mathfrak{A}(\mathcal{O})$ and the latter in terms of an abstract non-commutative version of Tannaka-Krein duality between an internal symmetry group $G$ and the representation category $R e p_{G}$ realized in the superselection sectors of $\mathfrak{A}$, respectively, and then combining two aspects in the form of the dynamical system $\mathfrak{F} \curvearrowleft G$. In the next section, the former aspect in relation with the scale changes is focused by means of the above net $\mathcal{O} \longmapsto \mathfrak{A}(\mathcal{O})$ of local observables. Concerning the problem of a symmetry breaking, what is to be in focus is the global aspects in which the differences of symmetries between internal and external become largely irrelevant.

With this understanding, we treat, as the algebra of the system under consideration, the $\mathrm{C}^{*}$-algebra $\mathfrak{F}$ of qunatum fields acted upon by a group $G$ which is supposed to represent all the possible kinds of symmetries characterizing the physical system. In the case of unbroken internal symmetry, the emerging group $G$ is verified to be a compact Lie group, whereas there is no guarantee of such a characterization of $G$ in the case of SSB. However, we assume here for technical reasons such a restriction on $G$ that it should be a locally compact group.

Now, the traditional treatment of SSB is just based on the so-called Goldstone commutators expressing the non-invariance $\omega(\delta(F)) \neq 0(\exists F \in \mathfrak{F})$ of 
a state $\omega$ under the infinitesimal transformations $\delta(\in \operatorname{Der}(\mathfrak{F})$ : densely defined *-derivations on $\mathfrak{F}$ ) in the directions of symmetry breaking; while this is a necessary condition for SSB in a pure (or more generally, factor) state, its sufficiency can be assured only for spatially homogeneous states such as vacuum ones. For instance, if factoriality of the representation is not required, one can easily obtain a $G$-invariant state even in the situation of SSB by averaging over a $G$-noninvariant factor state, which evades the necessity of $\omega(\delta(F)) \neq 0$. On the other hand, such a representation $(\pi, \mathfrak{H})$ can exist that in spite of the absence of $G$-invariant states in $\mathfrak{H}$ the symmetry is described by a unitary representation $G \ni g \longmapsto U(g) \in \mathcal{U}(\mathfrak{H})$ satisfying the so-called covariance condition $\pi\left(\tau_{g}(F)\right)=U(g) \pi(F) U(g)^{*}$. A general criterion for SSB can be given in the following form of definition so as to avoid these kinds of complications and to incorporate more general situations in an intrinsic way:

Definition 4.1 ([19]). A symmetry described by a strongly continuous automorphic action $\tau$ of a locally compact group $G$ on a (global) $\mathrm{C}^{*}$-algebra $\mathfrak{F}$ of quantum fields is said to be unbroken in a given representation $(\pi, \mathfrak{H})$ of $\mathfrak{F}$ if the spectrum of centre $\mathfrak{Z}_{\pi}(\mathfrak{F}):=\pi(\mathfrak{F})^{\prime \prime} \cap \pi(\mathfrak{F})^{\prime}$ is pointwise invariant ( $\mu$-a.e. w.r.t. the central measure $\mu$ for the central decomposition of $\pi$ into factor representations) under the action of $G$ induced on $\operatorname{Spec}\left(\mathfrak{Z}_{\pi}(\mathfrak{F})\right)$. If the symmetry is not unbroken in $(\pi, \mathfrak{H})$, it is said to be broken spontaneously there.

This definition exhibits the essence of SSB as the conflict between factoriality of $(\pi, \mathfrak{H})$ and unitary implementability of $G$ in it; in the usual approaches, the former point is respected at the expense of the latter. Taking the opposite choice to respect implementability we are led to the non-trivial centre which provides convenient tools for analyzing sector structure and flexible treatment of macroscopic order parameters to distinguish different sectors, as explained in the previous section: the central spectrum $\operatorname{Spec}\left(\mathfrak{Z}_{\pi}(\mathfrak{F})\right)$ physically means macroscopic order parameters appearing in low-energy infrared modes, and hence, the physical essence of this definition can be found in the "infrared instability" of the representation $(\pi, \mathfrak{H})$ along the direction of $G$-action which is in harmony with the intuitive physical picture of SSB.

Note, however, that this definition admits the coexistence of unbroken and broken subrepresentations in a given representation $\pi$ suffering from SSB, according to which the central spectrum $\operatorname{Spec}\left(\mathfrak{Z}_{\pi}(\mathfrak{F})\right)$ can further be decomposed into $G$-invariant subdomains. In view of the requirement of $G$-invariance, each such minimal subdomain is characterized by G-ergodicity, which means central ergodicity in the whole system. Therefore, $\pi$ is decomposed into the direct sum 
(or, direct integral) of unbroken factor representations and broken non-factor representations, each component of which is centrally $G$-ergodic. In this way we obtain a phase diagram on the spectrum of centre.

We can now construct a covariant representation of $(\mathfrak{F} \underset{\tau}{\curvearrowleft} G)$ implementing broken $G$ minimally in the sense of central G-ergodicity as follows:

1) Let $H$ be the maximal closed subgroup of $G$ unbroken in $(\pi, \mathfrak{H})$ with a covariant representation $(\pi, U, \mathfrak{H})$ of a $\mathrm{C}^{*}$-dynamical system $\mathfrak{F} \underset{\tau \uparrow_{H}}{\curvearrowleft} H$ satisfying $\pi\left(\tau_{h}(F)\right)=U(h) \pi(F) U(h)^{*}$ for $\forall h \in H$. An augmented algebra $\hat{\mathfrak{F}}:=\mathfrak{F} \rtimes$ $(H \backslash G)[20]$ is defined by a $\mathrm{C}^{*}$-crossed product of $\mathfrak{F}$ with the homogeneous space $H \backslash G$ which is realized as the algebra of continuous cross sections of $\mathrm{C}^{*}$-algebra bundle $G \times_{H} \mathfrak{F} \rightarrow H \backslash G$ :

$$
\hat{\mathfrak{F}}=\mathfrak{F} \rtimes(H \backslash G)=\Gamma\left(G \times_{H} \mathfrak{F}\right) .
$$

This can conveniently be identified with the algebra $C_{H}(G, \mathfrak{F})$ of $H$-equivariant continuous functions $\hat{F}$ on $G$ satisfying the condition

$$
\hat{F}(h g)=\tau_{h}(\hat{F}(g)) \quad \text { for } \forall g \in G, \forall h \in H .
$$

In what follows the identification of a cross section of $G \times_{H} \mathfrak{F} \rightarrow H \backslash G$ with an $H$-equivariant continuous function on $G$ is always understood and, without changing the notation, we freely move from one version to another. The product structure of $\hat{\mathfrak{F}}$ is simply given by the pointwise product,

$$
\left(\hat{F}_{1} \hat{F}_{2}\right)(\dot{g}):=\hat{F}_{1}(\dot{g}) \hat{F}_{2}(\dot{g}),
$$

for $\hat{F}_{1}, \hat{F}_{2} \in \hat{\mathfrak{F}}, \dot{g} \in H \backslash G$, which is equivalent to $\left(\hat{F}_{1} \hat{F}_{2}\right)(g):=\hat{F}_{1}(g) \hat{F}_{2}(g)$ in the version of $H$-equivariant continuous functions on $G$ consistently with the constraint of $H$-equivariance: $\left(\hat{F}_{1} \hat{F}_{2}\right)(h g)=\hat{F}_{1}(h g) \hat{F}_{2}(h g)=\tau_{h}\left(\hat{F}_{1}(g)\right) \tau_{h}\left(\hat{F}_{2}(g)\right)=$ $\tau_{h}\left(\left(\hat{F}_{1} \hat{F}_{2}\right)(g)\right)$. The action $\hat{\tau}$ of $G$ on $\hat{F} \in \hat{\mathfrak{F}}$ is defined by

$$
\left[\hat{\tau}_{g}(\hat{F})\right]\left(\dot{g}_{1}\right)=\hat{F}\left(\dot{g}_{1} g\right),
$$

or equivalently, $\left[\hat{\tau}_{g}(\hat{F})\right]\left(g_{1}\right)=\hat{F}\left(g_{1} g\right)$ for $H$-equivariant functions. The fixedpoint subalgebra $\hat{\mathfrak{F}}^{G}$ of $\hat{\mathfrak{F}}$ under the action $\hat{\tau}$ of $G$ is given by the constant section $\hat{F}: g \longmapsto F \in \mathfrak{F}^{H}$ because of the $H$-equivariance condition: $F=$ $\hat{F}(h g)=\tau_{h}(\hat{F}(g))=\tau_{h}(F)$ :

$$
\hat{\mathfrak{F}}^{G} \cong \mathfrak{F}^{H}
$$


Then from a representation $(\pi, U, \mathfrak{H})$ of a $\mathrm{C}^{*}$-dynamical system $\mathfrak{F} \underset{\tau \uparrow_{H}}{\curvearrowleft} H$, a representation $(\hat{\pi}, \hat{\mathfrak{H}})$ is induced of the crossed product $\hat{\mathfrak{F}}$ in the following way.

2) With the left-invariant Haar measure $d \xi$ on $G / H$ (with left $G$-action), the Hilbert space $\hat{\mathfrak{H}}$ is given by $L^{2}$-sections of $G \times_{H} \mathfrak{H}$ :

$$
\hat{\mathfrak{H}}=\int_{\xi \in G / H}^{\oplus}(d \xi)^{1 / 2} \mathfrak{H}=\Gamma_{L^{2}}\left(G \times_{H} \mathfrak{H}, d \xi\right),
$$

which can be identified with the $L^{2}$-space of $\mathfrak{H}$-valued $(U, H)$-equivariant functions $\psi$ on $G$,

$$
\psi(g h)=U\left(h^{-1}\right) \psi(g) \quad \text { for } \psi \in \hat{\mathfrak{H}}, g \in G, h \in H .
$$

On this $\hat{\mathfrak{H}}$, the representations $\hat{\pi}$ and $\hat{U}$ of $\hat{\mathfrak{F}}$ and $G$ are defined, respectively, by

$$
\begin{aligned}
(\hat{\pi}(\hat{F}) \psi)(g) & :=\pi\left(\hat{F}\left(g^{-1}\right)\right)(\psi(g)) \text { for } \hat{F} \in \hat{\mathfrak{F}}, \psi \in \hat{\mathfrak{H}}, g \in G, \\
\left(\hat{U}\left(g_{1}\right) \psi\right)(g) & :=\psi\left(g_{1}^{-1} g\right) \quad \text { for } g, g_{1} \in G,
\end{aligned}
$$

and satisfy the covariance relation:

$$
\hat{\pi}\left(\hat{\tau}_{g}(\hat{F})\right)=\hat{U}(g) \hat{\pi}(\hat{F}) \hat{U}(g)^{-1} .
$$

3) $\mathfrak{F}$ is embedded into $\hat{\mathfrak{F}}$ by $\hat{\imath}_{H \backslash G}: \mathfrak{F} \hookrightarrow \hat{\mathfrak{F}}$ given by

$$
\left[\hat{\imath}_{H \backslash G}(F)\right](g):=\tau_{g}(F),
$$

which is consistent with the $H$-equivariance condition: $\left[\hat{\imath}_{H \backslash G}(F)\right](h g)=\tau_{h g}(F)$ $=\tau_{h}\left(\left[\hat{\imath}_{H \backslash G}(F)\right](g)\right)$. This embedding map intertwines the $G$-actions $\tau$ on $\mathfrak{F}$ and $\hat{\tau}$ on $\hat{\mathfrak{F}}$,

$$
\hat{\imath}_{H \backslash G} \circ \tau_{g}=\hat{\tau}_{g} \circ \hat{\imath}_{H \backslash G} \quad(\forall g \in G),
$$

and hence, we have

$$
\left[\hat{\imath}_{H \backslash G}(\mathfrak{F})\right]^{G}=\hat{\imath}_{H \backslash G}\left(\mathfrak{F}^{G}\right) \subset \hat{\imath}_{H \backslash G}\left(\mathfrak{F}^{H}\right)=\hat{\mathfrak{F}}^{G} .
$$

The mutual relations among (sub)algebras and mappings can be depicted by

$$
\begin{aligned}
& \hat{\mathfrak{F}}=\Gamma(\underset{H}{\times \mathfrak{F}}) \\
& \hat{\imath}_{G} \nearrow \swarrow \hat{m}_{G} \\
& \mathfrak{F}^{H} \cong \hat{\imath}_{H \backslash G}\left(\mathfrak{F}^{H}\right)=\hat{\mathfrak{F}}^{G} \quad \underset{m_{H}}{\stackrel{i_{H}}{\rightleftarrows}} \\
& i_{G / H} \nwarrow \searrow m_{G / H} \\
& \mathfrak{F}^{G}
\end{aligned}
$$

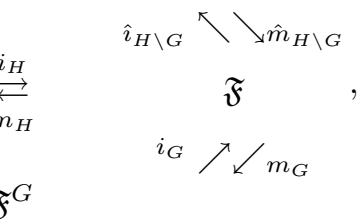


where the maps $i_{G}$ and $m_{G}$, etc., are, respectively, the embedding maps (of a $\mathrm{C}^{*}$-algebra into another) and the conditional expectations defined as operatorvalued weights to extract fixed points, such as

$$
m_{G / H}: \mathfrak{F}^{H} \ni B \longmapsto m_{G / H}(B):=\int_{G / H} d \dot{g} \tau_{g}(B) \in \mathfrak{F}^{G} .
$$

4) Combining $\hat{\imath}_{H \backslash G}$ with $\hat{\pi}$, we obtain a covariant representation $(\bar{\pi}, \hat{U}, \hat{\mathfrak{H}})$, $\bar{\pi}:=\hat{\pi} \circ \hat{\imath}_{H \backslash G}$, of $\mathfrak{F} \underset{\tau}{\curvearrowleft} G$ defined on $\hat{\mathfrak{H}}$ by

$$
(\bar{\pi}(F) \psi)(g):=\pi\left(\tau_{g^{-1}}(F)\right) \psi(g) \quad(F \in \mathfrak{F}, \psi \in \hat{\mathfrak{H}})
$$

and satisfying

$$
\bar{\pi}\left(\tau_{g}(F)\right)=\hat{U}(g) \bar{\pi}(F) \hat{U}(g)^{-1} .
$$

5) The sector structure is determined by the following information on the relevant centres:

Proposition 4.1. When the von Neumann algebra $\pi(\mathfrak{F})^{\prime \prime}$ has a trivial centre, $\mathfrak{Z}_{\pi}(\mathfrak{F})=\mathbb{C} 1$, the centres of $\hat{\pi}(\hat{\mathfrak{F}})^{\prime \prime}$ and $\bar{\pi}(\mathfrak{F})^{\prime \prime}$ are given by

$$
\mathfrak{Z}_{\bar{\pi}}(\mathfrak{F})=L^{\infty}(H \backslash G ; d \dot{g})=\mathfrak{Z}_{\hat{\pi}}(\hat{\mathfrak{F}}) .
$$

This can be seen as follows: From the definition of $\hat{\mathfrak{F}} \subset C(H \backslash G, \mathfrak{F}) \cong$ $C(H \backslash G) \bar{\otimes} \mathfrak{F}$ it is clear that the commutative algebra $C(H \backslash G)$ is contained in the centre of the $\mathrm{C}^{*}$-algebra $\hat{\mathfrak{F}}$. If this centre is bigger than $C(H \backslash G)$, it contains a function $\hat{F}$ on $H \backslash G$ whose image $\hat{F}(\dot{g})$ at some point $\dot{g} \in H \backslash G$ is not be a scalar multiple of the identity, which does not commute with some element $F_{1} \in \mathfrak{F}$ because of the triviality of the centre of $\mathfrak{F}$ due to $\mathfrak{Z}_{\pi}(\mathfrak{F})=\mathbb{C} 1$ : $\left[\hat{F}(\dot{g}), F_{1}\right] \neq 0$.

In view of 3$), F_{1}$ can be embedded in $\hat{\mathfrak{F}}$ satisfying $\hat{\imath}_{H \backslash G}\left(F_{1}\right)(\dot{e})=F_{1}$, and hence, we have $\hat{\tau}_{g^{-1}} \hat{\imath}_{H \backslash G}\left(F_{1}\right)(\dot{g})=F_{1}$, which shows the relation $[\hat{F}$, $\left.\hat{\tau}_{g^{-1}} \hat{\imath}_{H \backslash G}\left(F_{1}\right)\right](\dot{g})=\left[\hat{F}(\dot{g}), F_{1}\right] \neq 0$. Thus, we have $\mathfrak{Z}(\hat{\mathfrak{F}})=C(H \backslash G)$. Using the similar arguments for $\hat{\pi}(\hat{\mathfrak{F}})^{\prime \prime}$ combined with $\mathfrak{Z}_{\bar{\pi}}(\mathfrak{F}) \subset L^{\infty}(H \backslash G, d \dot{g}) \bar{\otimes} \pi(\mathfrak{F})^{\prime \prime}$, we see $\mathfrak{Z}_{\hat{\pi}}(\hat{\mathfrak{F}})=L^{\infty}(H \backslash G ; d \dot{g})$. The equality $\mathfrak{Z}_{\bar{\pi}}(\mathfrak{F})=L^{\infty}(H \backslash G ; d \dot{g})$ comes from the mutual disjointness $\pi \circ$ ' $\left(\pi \circ \tau_{g}\right)$ for $g \in G \backslash H$ and $\mathfrak{Z}_{\pi}(\mathfrak{F})=\mathbb{C} 1$.

Since the homogeneous space $H \backslash G$ as the spectrum of the centre $\mathfrak{Z}_{\bar{\pi}}(\mathfrak{F})$ is transitive under the right action of $G$ which is just the action induced on the central spectrum from $\hat{\tau}$, the representation $(\bar{\pi}, \hat{\mathfrak{H}})$ of the dynamical system $\mathfrak{F} \underset{\tau}{\curvearrowleft} G$ is centrally $G$-ergodic, to which the criterion for SSB can be applied. 
Adapting the above formulation to the GNS representation $\left(\pi=\pi_{\beta}, \mathfrak{H}=\right.$ $\left.\mathfrak{H}_{\beta}\right)$ of a KMS state $\omega_{\beta=\left(\beta^{\mu}\right)}$ with $H=\mathbb{R}^{4} \rtimes S O(3), G=\mathbb{R}^{4} \rtimes L_{+}^{\uparrow}$, we can reproduce the results on the SSB of Lorentz boosts [16]: $\mathfrak{Z}_{\bar{\pi}}(\mathfrak{F})=L^{\infty}\left(S O(3) \backslash L_{+}^{\uparrow}\right)=$ $L^{\infty}\left(\mathbb{R}^{3}\right)$ through the identification $\beta^{\mu} / \sqrt{\beta^{2}}=u^{\mu}=\left(\frac{1}{\sqrt{1-\mathbf{v}^{2} / c^{2}}}, \frac{\mathbf{v}}{\sqrt{1-\mathbf{v}^{2} / c^{2}}}\right) \longleftrightarrow$ $\mathbf{v} \in \mathbb{R}^{3}$.

\section{$\S 5 . \quad H o w$ to Formulate Broken Scale Invariance?}

As noted at the beginning, the above discussion of symmetry breakdown was concerning the spontaneous breakdown of a symmetry described by a group $G$ acting on the field algebra $\mathfrak{F}$ by automorphisms. In contrast, the notion of the broken scale invariance is usually understood in a physical system with such explicit breaking terms as non-vanishing mass, which seem to cause difficulties in treating scale transformations as automorphisms acting on the algebra describing the system. However, the results on the scaling algebra in algebraic QFT due to Buchholz and Verch [6] shows that the above negative anticipation can safely be avoided.

Their results can be summarized as follows. Let the following requirements be imposed on all the possible renormalization-group transformations $R_{\lambda}$ :

(i) $R_{\lambda}$ should map the given net $\mathcal{O} \rightarrow \mathfrak{A}(\mathcal{O})$ of local observables at spacetime scale 1 onto the corresponding net $\mathcal{O} \rightarrow \mathfrak{A}_{\lambda}(\mathcal{O}):=\mathfrak{A}(\lambda \mathcal{O})$ at a scale $\lambda$, i.e.,

$$
R_{\lambda}: \mathfrak{A}(\mathcal{O}) \rightarrow \mathfrak{A}_{\lambda}(\mathcal{O})
$$

for every region $\mathcal{O} \subset \mathbb{R}^{4}$. Since both time and space are scaled by the same $\lambda$, the light velocity $c$ remains unchanged as their ratio.

(ii) In the Fourier-transformed picture, the subspace $\widetilde{\mathfrak{A}}(\widetilde{\mathcal{O}})$ of all (quasilocal) observables carrying energy-momentum in the set $\widetilde{\mathcal{O}} \subset \mathbb{R}^{4}$ is transformed as

$$
R_{\lambda}: \widetilde{\mathfrak{A}}(\widetilde{\mathcal{O}}) \rightarrow \widetilde{\mathfrak{A}}_{\lambda}(\widetilde{\mathcal{O}})
$$

where $\widetilde{\mathfrak{A}}_{\lambda}(\widetilde{\mathcal{O}}):=\widetilde{\mathfrak{A}}\left(\lambda^{-1} \widetilde{\mathcal{O}}\right)$. In view of the duality between spacetime coordinates $x^{\mu}$ and energy-momenum $p_{\mu}$ involved in the Fourier transformation, this requirement implies the invariance of the quantity $p_{\mu} x^{\mu}=E t-\vec{p} \cdot \vec{x}$ called "action" in physics, as a consequence of which the Planck constant $\hbar$ with the dimension of action also remains invariant.

(iii) $R_{\lambda}$ should be bounded continuous maps uniformly in $\lambda$ (even if they may not be isomorphisms): concerning the possibility of non-isomorphisms, 
Buchholz and Verch in [6] remark "In the case of dilation invariant theories the transformations $R_{\lambda}$ are expected to be isomorphisms, yet this will not be true in general since the algebraic relations between observables may depend on the scale." In contrast to their focus on the high-energy limits in the context of vacuum situations, our interest here is in the thermal situations involving all the possible temperatures. But the similar point to the scale dependence of the basic algebraic relations should be expected to show up especially in the direction to the low temperature side, because of the increasing complexity of phase structures arising from the bifurcating processes of phase transitions. In view of the seemingly ad hoc choices of the starting dynamics and the algebras of relevant physical variables in the standard approaches to phase transitions, it should be certainly one of the non-trivial important questions whether all the variety of different thermodynamic phases can be totally attributed to that of the realized states and representations of one and the same fixed dynamical system with a fixed algebra of observables and a fixed dynamics acting on the former.

Then, the scaling net $\mathcal{O} \rightarrow \hat{\mathfrak{A}}(\mathcal{O})$ corresponding to the original local net $\mathcal{O} \rightarrow \mathfrak{A}(\mathcal{O})$ of observables is defined as the local net consisting of scale-changed observables under the action of all the possible choice of $R_{\lambda}$ satisfying (i)(iii). The results on the structure of $\hat{\mathfrak{A}}(\mathcal{O})$ obtained in [6] can be reformulated into the identification of $\hat{\mathfrak{A}}(\mathcal{O})$ with the algebra $\Gamma\left(\amalg_{\lambda \in \mathbb{R}^{+}} \mathfrak{A}_{\lambda}(\mathcal{O})\right)$ of sections $\mathbb{R}^{+} \ni \lambda \longmapsto \hat{A}(\lambda) \in \mathfrak{A}_{\lambda}(\mathcal{O})$ of algebra bundle $\amalg_{\lambda \in \mathbb{R}^{+}} \mathfrak{A}_{\lambda}(\mathcal{O}) \rightarrow \mathbb{R}^{+}$over the multiplicative group $\mathbb{R}^{+}$of scale changes:

$$
\hat{\mathfrak{A}}(\mathcal{O})=\Gamma\left(\amalg_{\lambda \in \mathbb{R}^{+}} \mathfrak{A}_{\lambda}(\mathcal{O})\right) \ni \hat{A}:=\left(\mathbb{R}^{+} \ni \lambda \longmapsto \hat{A}(\lambda) \in \mathfrak{A}_{\lambda}(\mathcal{O})\right) .
$$

The scaling algebra $\hat{\mathfrak{A}}$ playing the role of the global algebra is defined by the $\mathrm{C}^{*}$-inductive limit of all local algebras $\hat{\mathfrak{A}}(\mathcal{O})$ on the basis of the isotony $\hat{\mathfrak{A}}\left(\mathcal{O}_{1}\right) \subset \hat{\mathfrak{A}}\left(\mathcal{O}_{2}\right)$ for $\mathcal{O}_{1} \subset \mathcal{O}_{2}$.

The algebraic structures to make $\hat{\mathfrak{A}}(\mathcal{O})$ a unital $\mathrm{C}^{*}$-algebra are defined in a pointwise manner by

$$
\begin{aligned}
& \left(c_{1} \hat{A}_{1}+c_{2} \hat{A}_{2}\right)(\lambda):=c_{1} \hat{A}_{1}(\lambda)+c_{2} \hat{A}_{2}(\lambda), \\
& \left(\hat{A}_{1} \hat{A}_{2}\right)(\lambda):=\hat{A}_{1}(\lambda) \hat{A}_{2}(\lambda), \\
& \hat{\mathbf{1}}(\lambda):=\mathbf{1}=\mathbf{1}_{\mathfrak{A}}, \\
& \left(\hat{A}^{*}\right)(\lambda):=\hat{A}(\lambda)^{*},
\end{aligned}
$$

(for $\hat{A}_{1}, \hat{A}_{2}, \hat{A} \in \hat{\mathfrak{A}}(\mathcal{O}), c_{1}, c_{2} \in \mathbb{C}$ ) and the $\mathrm{C}^{*}$-norm by

$$
\|\hat{A}\|:=\sup _{\lambda \in \mathbb{R}^{+}}\|\hat{A}(\lambda)\| .
$$


From the scaled actions $\mathfrak{A}_{\lambda} \underset{\alpha^{(\lambda)}}{\curvearrowleft} \mathcal{P}_{+}^{\uparrow}$ of the Poincaré group on $\mathfrak{A}_{\lambda}$ with $\alpha_{a, \Lambda}^{(\lambda)}=$ $\alpha_{\lambda a, \Lambda}$, an action of $\mathcal{P}_{+}^{\uparrow}$ is induced on $\hat{\mathfrak{A}}$ by

$$
\left(\hat{\alpha}_{a, \Lambda}(\hat{A})\right)(\lambda):=\alpha_{\lambda a, \Lambda}(\hat{A}(\lambda)),
$$

in terms of which the essence of the condition (iii) is expressed simply as the continuity of the action of the Poincaré group at its identity: $\| \hat{\alpha}_{a, \Lambda}(\hat{A})-$ $\hat{A} \| \underset{(a, \Lambda) \rightarrow(0,1)}{\longrightarrow} 0$. The so-defined scaling net $\mathcal{O} \rightarrow \hat{\mathfrak{A}}(\mathcal{O})$ is shown to satisfy all the properties to characterize a relativistic local net of observables if the original one $\mathcal{O} \rightarrow \mathfrak{A}(\mathcal{O})$ does.

Then, the scale transformation is defined by an automorphic action $\hat{\sigma}$ of the group $\mathbb{R}^{+}$of scale changes on the scaling algebra $\hat{\mathfrak{A}}$ given for $\forall \mu \in \mathbb{R}^{+}$by

$$
\left(\hat{\sigma}_{\mu}(\hat{A})\right)(\lambda):=\hat{A}(\mu \lambda), \quad \lambda>0,
$$

satisfying the properties:

$$
\begin{aligned}
\hat{\sigma}_{\mu}(\hat{\mathfrak{A}}(\mathcal{O})) & =\hat{\mathfrak{A}}(\mu \mathcal{O}), \quad \mathcal{O} \subset \mathbb{R}^{4}, \\
\hat{\sigma}_{\mu} \circ \hat{\alpha}_{a, \Lambda} & =\hat{\alpha}_{\mu a, \Lambda} \circ \hat{\sigma}_{\mu}, \quad(a, \Lambda) \in \mathcal{P}_{+}^{\uparrow} .
\end{aligned}
$$

In this formulation, the roles of renormalization-group transformations to relate observables at different scales are played by the scaling transformations $\hat{\sigma}_{\mathbb{R}^{+}}$acting isomorphically on the scaling net $\mathcal{O} \rightarrow \hat{\mathfrak{A}}(\mathcal{O})$. In view of the algebra $C\left(\mathbb{R}^{+}\right)$of scalar-valued functions on $\mathbb{R}^{+}$embedded in the centre of the scaling algebra $\hat{\mathfrak{A}}, C\left(\mathbb{R}^{+}\right) \hookrightarrow \mathfrak{Z}(\hat{\mathfrak{A}}) \subset \hat{\mathfrak{A}}$, it is no miracle for a broken scale invariance caused by such explicit breaking terms as the mass $m$ to be restored as an "exact" symmetry described by an automorphic action $\hat{\sigma}$ of $\mathbb{R}^{+}$when all the terms responsible for the explicit breaking can be treated (as is common in practice) in terms of scale-dependent classical variables like $\mathbb{R}^{+} \ni \lambda \longmapsto m(\lambda)=\lambda^{d_{m}} m_{0}$. It is also remarkable that the final results obtained by Buchholz and Verch [6] through the complicated analysis can naturally be seen just as a special case of the previous definition of the augmented algebra $\hat{\mathfrak{F}}:=\Gamma\left(G \times_{H} \mathfrak{F}\right)$ for treating SSB with the choice of $H:=\mathcal{P}_{+}^{\uparrow}, G=H \rtimes \mathbb{R}^{+}$(semidirect product of groups) in combination with a slight modification due to the spacetime dependence described by the local net structure: $\mathfrak{F} \Longrightarrow(\mathcal{O} \rightarrow \mathfrak{A}(\mathcal{O})$ ) (upon which the group $\mathbb{R}^{+}$of scale changes acts). While this is the case found in the vacuum situations which show the invariance under the Poincaré group $\mathcal{P}_{+}^{\uparrow}=\mathbb{R}^{4} \rtimes L_{+}^{\uparrow}$ by definition, the typical thermal situations relevant to our present contexts require more careful treatment because of the SSB of Lorentz 
boost symmetry caused by temperatures. Here the Poincaré group $\mathcal{P}_{+}^{\uparrow}$ of relativistic symmetry is broken down to $\mathbb{R}^{4} \rtimes S O(3)$ and, in the opposite direction, it is extended to a larger one $\mathcal{P}_{+}^{\uparrow} \rtimes \mathbb{R}^{+}$involving the broken scale invariance, which may possibly be extended at the critical points further to the conformal group $S O(2,4)$. It is interesting to note that the series of group extensions $S O(3) \hookrightarrow S O(1,3) \hookrightarrow S O(1,3) \rtimes \mathbb{R}^{+} \hookrightarrow S O(2,4)$ (or its doube covering $\left.S U(2) \hookrightarrow S L(2, \mathbb{C}) \hookrightarrow S L(2, \mathbb{C}) \rtimes \mathbb{R}^{+} \hookrightarrow S U(2,2)\right)$ can be understood in the context of the Kantor-Koecher-Tits construction of Lie algebras associated with a symmetric space and the corresponding Jordan triple system [23], the last member of which gives the analytic group of automorphisms of the tube domain $\mathbb{R}^{4}+i V_{+} \ni x^{\mu}+i \beta^{\mu}$. If we start from the choice of $H:=\mathcal{P}_{+}^{\uparrow}, G=H \rtimes \mathbb{R}^{+}$ even in the thermal situation at $T \neq 0 K$, the starting representation $(\pi, \mathfrak{H})$ with $H$ as the group of unbroken symmetry should be understood to contain already a non-trivial centre with $S O(3) \backslash L_{+}^{\uparrow} \cong \mathbb{R}^{3}$ as its spectrum due to the $\mathrm{SSB}$ of $\mathcal{P}_{+}^{\uparrow}$ down to $\mathbb{R}^{4} \rtimes S O(3)$. In this context, the scaled actions $\alpha_{a, \Lambda}^{(\lambda)}=\alpha_{\lambda a, \Lambda}$ of Poincaré group on $\mathfrak{A}_{\lambda}$ can be naturally understood as the conjugacy change of the stability group $H \rightarrow g H g^{-1}$ from the point $H e$ to $H^{-1}$ on the base space $H \backslash G=\mathbb{R}^{+}: s_{\lambda}(a, \Lambda) s_{\lambda}^{-1}=(\lambda a, \Lambda)$, where $s_{\lambda}\left(x^{\mu}\right)=\lambda x^{\mu}$.

\section{$\S 6 . \quad$ Scale Changes on States}

In relation with the centre $\mathfrak{Z}(\hat{\mathfrak{A}})=\mathfrak{Z}(\hat{\mathfrak{A}}(\mathcal{O}))=C\left(\mathbb{R}^{+}\right)$arising from the broken scale invariance, we have a canonical family of conditional expectations $\hat{\mu}$ from $\hat{\mathfrak{A}}$ to $\mathfrak{A}$ corresponding to probability measures $\mu$ on $C\left(\mathbb{R}^{+}\right)$:

$$
\hat{\mu}: \hat{\mathfrak{A}} \ni \hat{A} \longmapsto \int_{\mathbb{R}^{+}} d \mu(\lambda) \hat{A}(\lambda) \in \mathfrak{A} .
$$

(We can consider the case with $d \mu(\lambda)$ chosen to be the Haar measure $d \lambda / \lambda$ of $\mathbb{R}^{+}$, which is, however, a positive unbounded measure but not a probability one; the corresponding $\hat{\mu}$ becomes then an operator-valued weight whose images are not guaranteed to be finite.) By means of $\hat{\mu}$, any state $\omega \in E_{\mathfrak{A}}$ can be lifted onto $\hat{\mathfrak{A}}$ by

$$
E_{\mathfrak{A}} \ni \omega \longmapsto \hat{\mu}^{*}(\omega)=\omega \circ \hat{\mu}=\omega \otimes \mu \in E_{\hat{\mathfrak{A}}},
$$

where we have used $\hat{\mathfrak{A}} \subset C\left(\mathbb{R}^{+}, \mathfrak{A}\right) \cong \mathfrak{A} \otimes C\left(\mathbb{R}^{+}\right)$.

In [6] the case of $\mu=\delta_{\lambda=1}\left(\right.$ : Dirac measure at the identity of $\left.\mathbb{R}^{+}\right)$is called a canonical lift $\hat{\omega}:=\omega \circ \hat{\delta}_{1}$. The scale transformed state defined by

$$
\hat{\omega}_{\lambda}:=\hat{\omega} \circ \hat{\sigma}_{\lambda}=\omega \circ \hat{\delta}_{\lambda}
$$


describes the situation at scale $\lambda$ due to the renormalization-group transformation of scale change $\lambda$.

Conversely, starting from a state $\hat{\omega}$ of $\hat{\mathfrak{A}}$, we can obtain its central decomposition as follows: first, we call two natural embedding maps $\iota: \mathfrak{A} \hookrightarrow \hat{\mathfrak{A}}$ $[[\iota(A)](\lambda) \equiv A]$ and $\kappa: C\left(\mathbb{R}^{+}\right) \simeq \mathfrak{Z}(\hat{\mathfrak{A}}) \hookrightarrow \hat{\mathfrak{A}}$. Pulling back $\hat{\omega}$ by $\kappa^{*}: E_{\hat{\mathfrak{A}}} \rightarrow$ $E_{C\left(\mathbb{R}^{+}\right)}$, we can define a probability measure $\rho_{\hat{\omega}}:=\kappa^{*}(\hat{\omega})=\hat{\omega} \circ \kappa=\hat{\omega}\left\lceil_{C\left(\mathbb{R}^{+}\right)}\right.$ on $\mathbb{R}^{+}$, namely, $\hat{\omega}\left\lceil_{C\left(\mathbb{R}^{+}\right)}(f)=\int_{\mathbb{R}^{+}} d \rho_{\hat{\omega}}(\lambda) f(\lambda)\right.$ for $\forall f \in C\left(\mathbb{R}^{+}\right)$.

For any positive operator $\hat{A}=\int a d \hat{E}_{\hat{A}}(a) \in \hat{\mathfrak{A}}$, we can consider the central $\operatorname{supports} c\left(\hat{E}_{\hat{A}}(\Delta)\right) \in \operatorname{Proj}\left(\mathfrak{Z}_{\hat{\pi}_{\hat{\omega}}}(\hat{\mathfrak{A}})\right)$ of $\hat{E}_{\hat{A}}(\Delta) \in \operatorname{Proj}\left(\hat{\pi}_{\hat{\omega}}(\hat{\mathfrak{A}})^{\prime \prime}\right)$ with a Borel set $\Delta$ in $S p(\hat{A}) \subset[0,+\infty)$ satisfying $c\left(\hat{E}_{\hat{A}}(\Delta)\right) \hat{E}_{\hat{A}}(\Delta)=\hat{E}_{\hat{A}}(\Delta)$, from which we see that $\rho_{\hat{\omega}}^{\prime \prime}\left(c\left(\hat{E}_{\hat{A}}(\Delta)\right)\right)=0$ implies $\hat{\omega}^{\prime \prime}\left(\hat{E}_{\hat{A}}(\Delta)\right)=0$, where $\hat{\omega}^{\prime \prime}$ and $\rho_{\hat{\omega}}^{\prime \prime}$ are the extensions of $\hat{\omega}$ and $\rho_{\hat{\omega}}$ to $\hat{\pi}_{\hat{\omega}}(\hat{\mathfrak{A}})^{\prime \prime}$ and $L^{\infty}\left(\mathbb{R}^{+}, d \rho_{\hat{\omega}}\right)$, respectively. Thus, we can define the Radon-Nikodym derivative $\omega_{\lambda}:=\frac{d \hat{\omega}}{d \rho_{\hat{\omega}}}(\lambda)$ of $\hat{\omega}$ w.r.t. $\rho_{\hat{\omega}}$ as a state on $\hat{\pi}_{\hat{\omega}}(\hat{\mathfrak{A}})^{\prime \prime}$ (in a similar way to [22]) so that

$$
\hat{\omega}(\hat{A})=\int d \rho_{\hat{\omega}}(\lambda) \omega_{\lambda}(\hat{A}(\lambda))=\int d \rho_{\hat{\omega}}(\lambda) \omega_{\lambda}\left(\hat{\delta}_{\lambda}(\hat{A})\right)=\int d \rho_{\hat{\omega}}(\lambda)\left[\omega_{\lambda} \otimes \hat{\delta}_{\lambda}\right](\hat{A}) .
$$

Then, the pull-back $\iota^{*}(\hat{\omega})=\hat{\omega} \circ \iota \in E_{\mathfrak{A}}$ of $\hat{\omega} \in E_{\hat{\mathfrak{A}}}$ by $\iota^{*}: E_{\hat{\mathfrak{A}}} \rightarrow E_{\mathfrak{A}}$ is given by

$$
\iota^{*}(\hat{\omega})=\int d \rho_{\hat{\omega}}(\lambda) \omega_{\lambda},
$$

owing to the relation

$$
\iota^{*}(\hat{\omega})(A)=\hat{\omega}(\iota(A))=\int d \rho_{\hat{\omega}}(\lambda) \omega_{\lambda}(A)=\left[\int d \rho_{\hat{\omega}}(\lambda) \omega_{\lambda}\right](A) .
$$

Applying this relation to the scaled canonical lift, $\hat{\omega}_{\lambda}:=\hat{\omega} \circ \hat{\sigma}_{\lambda}=\left(\omega \circ \hat{\delta}_{1}\right) \circ \hat{\sigma}_{\lambda}=$ $\omega \circ \hat{\delta}_{\lambda}$, of a state $\omega \in E_{\mathfrak{A}}$, we can easily see $\iota^{*}\left(\omega \circ \hat{\delta}_{\lambda}\right)=\iota^{*}\left(\hat{\omega}_{\lambda}\right)=\omega_{\lambda}[=$ $\left.\frac{d \hat{\omega}_{\lambda}}{d \delta_{\lambda}}(\lambda)\right]=\phi_{\lambda}(\omega)$, where $\phi_{\lambda}$ is the isomorphism introduced in [6] between $\omega$ and the canonical lift $\hat{\omega}_{\lambda} \in E_{\hat{\mathfrak{A}}}$ projected onto $\hat{\mathfrak{A}} / \operatorname{ker}\left(\hat{\pi}_{\hat{\omega}} \circ \hat{\sigma}_{\lambda}\right)$.

Thus, we can lift canonically any state $\omega \in E_{\mathfrak{A}}$ from $\mathfrak{A}$ to $\hat{\omega} \in E_{\hat{\mathfrak{A}}}$, and, after the scale shift $\hat{\sigma}_{\lambda}$ on $\hat{\mathfrak{A}}$, return $\hat{\omega} \circ \hat{\sigma}_{\lambda}$ back onto $\mathfrak{A}: \phi_{\lambda}(\omega)=\omega_{\lambda}=\iota^{*}\left(\omega \circ \hat{\delta}_{\lambda}\right)$, as result of which we obtain the scale-shifted state $\omega_{\lambda} \in E_{\mathfrak{A}}$ from $\omega \in E_{\mathfrak{A}}$ in spite of the absence of scale invariance on $\mathfrak{A}$.

Now applying this procedure to $\omega=\omega_{\beta}$ (: any state belonging to the family of relativistic KMS states with the same $\left.\left(\beta^{2}\right)^{1 / 2}\right)$, we have a genuine KMS state by going to their rest frames. Then we have $\hat{\omega}_{\lambda}=\left(\widehat{\omega_{\beta}}\right)_{\lambda}=\omega_{\beta} \circ \widehat{\delta_{\lambda}}$ 
which is shown to be a KMS state at $\beta / \lambda$ :

$$
\begin{aligned}
\left(\omega_{\beta} \circ \widehat{\delta_{\lambda}}\right)\left(\hat{A} \hat{\alpha}_{t}(\hat{B})\right) & =\omega_{\beta}\left(\hat{A}(\lambda) \alpha_{\lambda t}(\hat{B}(\lambda))\right) \\
=\omega_{\beta}\left(\alpha_{\lambda t-i \beta}(\hat{B}(\lambda)) \hat{A}(\lambda)\right) & =\omega_{\beta}\left(\alpha_{\lambda(t-i \beta / \lambda)}(\hat{B}(\lambda)) \hat{A}(\lambda)\right) \\
& =\left(\omega_{\beta} \circ \widehat{\delta_{\lambda}}\right)\left(\hat{\alpha}_{t-i \beta / \lambda}(\hat{B}) \hat{A}\right),
\end{aligned}
$$

and hence, $\left(\widehat{\omega_{\beta}}\right)_{\lambda} \in \hat{K}_{\beta / \lambda}, \phi_{\lambda}\left(\omega_{\beta}\right) \in K_{\beta / \lambda}$.

As has been already remarked, the above discussion is seen to apply equally to the spontaneous as well as explicitly broken scale invariance with such explicit breaking parameters as mass terms. The actions of scale transformations on such variables as $x^{\mu}, \beta^{\mu}$ and also conserved charges are just straightforward, which is justified by such facts that the first and the second ones are of kinematical nature and that the second and the third ones exhibit themselves in the state labels for specifying the relevant sectors in the context of the superselection structures $[5,20]$. This gives an alternative verification to the so-called non-renormalization theorem of conserved charges. In sharp contrast, other such variables as coupling constants (to be read off from the data of correlation functions or Green's functions) are affected by the scaled dynamics, and hence, may show non-trivial scaling behaviours with deviations from the canonical (or kinematical) dimensions, in such forms as the running couplings or anomalous dimensions. Thus, the transformations $\hat{\sigma}_{\lambda}$ (as "exact" symmetry on the augmented algebra $\hat{\mathfrak{A}}$ ) are understood to play the roles of the renormalization-group transformations (as broken symmetry on the original algebra $\mathfrak{A}$ ).

As a result, we see that classical macroscopic observable $\beta$ naturally emerging from a microscopic quantum system is verified to be an order parameter of broken scale invariance involved in the renormalization group in relativistic QFT.

\section{$\S 7 . \quad$ Summary and Outlook}

To equip such expressions as "broken scale invariance" and its "order parameter" with their precise formulations, we have adopted a scheme to incorporate spontaneously as well as explicitly broken symmetries with the criterion for symmetry breakdown on the basis of an augmented algebra with a non-trivial centre in such forms as $\hat{\mathfrak{F}}=\Gamma\left(G \times_{H} \mathfrak{F}\right)$ or $\mathcal{O} \longmapsto \hat{\mathfrak{A}}(\mathcal{O})=\Gamma\left(\amalg_{\lambda \in \mathbb{R}^{+}} \mathfrak{A}(\lambda \mathcal{O})\right)$, the latter of which is just a re-interpretation of the Buchholz-Verch scaling net of local observables adapted to the former. While the general scheme itself explained in item 3) of Sec.3 is unchanged (whose components may change, though) by the presence of explicitly broken symmetries, the criterion for symmetry breaking (Definition 7 in Sec.4) in this case should naturally be understood with 
$\mathfrak{F}$ replaced by the augmented one. As an algebra of the composite system of a genuine quantum one together with classical macroscopic one (embedded as the centre), the augmented algebra $\hat{\mathfrak{F}}$ or $\hat{\mathfrak{A}}$ can play such important roles that

a) it allows a symmetry explicitly broken by breaking terms (such as nonvanishing masses in the case of scale invariance) to be formulated in terms of the symmetry transformations acting on $\hat{\mathfrak{A}}$ through automorphisms $\epsilon$ $A u t(\hat{\mathfrak{A}})$ which is realized by the simultaneous changes of the breaking terms belonging to the centre $\mathfrak{Z}(\hat{\mathfrak{A}})$ to cancel the breaking effects,

a') for a spontaneously broken symmetry, this augmented algebra naturally accommodates its covariant unitary representation as an induced representation from a subgroup of the remaining unbroken symmetry (at the expense of the non-trivial centre characterizing the symmetry breaking),

b) the continuous behaviours of order parameters under the broken symmetry transformations is algebraically expressed at the $\mathrm{C}^{*}$-level of the $\left(\mathrm{C}^{*}\right.$ algebraic) centre $\mathfrak{Z}(\hat{\mathfrak{A}})$ in sharp contrast to the discontinuous ones at the $\mathrm{W}^{*}$-level $\mathfrak{Z}_{\pi}(\hat{\mathfrak{A}})$ of representations owing to the mutual disjointness among representations corresponding to different values of order parameters (as points on $\operatorname{Spec}\left(\mathfrak{Z}_{\pi}(\mathfrak{A})\right)$ ). To this continuous order parameter some external field can further be coupled, like the coupling between the magnetization and an external magnetic field in the discussion of a Heisenberg ferromagnet. Using this coupling, we can examine, for instance, the mutual relations between the magnetization caused by an external field and the spontaneous one, the latter of which persists in the asymptotic removal of the former in combination with the hysteresis effects. Without introducing the augmented algebra $\hat{\mathfrak{A}}$, it would be difficult for this kind of discussions to be adapted to the case of QFT.

Then, the mutual relation between states on $\mathfrak{A}$ and $\hat{\mathfrak{A}}$ is clarified, on the basis of which the verification of the statement on the behaviour of the inverse temperatures is just reduced to a simple computation of checking the parameter shift occurring in the KMS condition under the scale transformation. What is interesting in this observation about the roles of the (inverse) temperature $\beta$ is that it exhibits the cross over between thermal and geometric aspects expressed in $\beta^{\mu}=\beta u^{\mu}$ and in the spacetime transformations $\mathcal{P}_{+}^{\uparrow} \rtimes \mathbb{R}^{+}$ including the scale one, respectively. We note also that the introduction of augmented algebras allows us to get a free control, through different combinations of scale transformations (of $t, \vec{x}, E, \vec{p}$, etc.), over the size of such constants 
as not only mass $m$ but also any physical constants (method of variation of physical constants [21]) like Planck constant $\hbar$, light velocity $c$, Boltzmann constant $k_{B}$, gauge and gravitational couplings $e, \kappa$, etc., which becomes relevant to undoing the unnecessary changes in unfocused constants to be fixed at the original values. With this freedom at hand, we can expect realistic possibilities of comparisons and controls over mutual shifts among theories and branches in physics describing different domains and aspects in nature, in terms of selection criteria and of these constants to characterize each of such theories.

While the above scale transformations in the real version cause actual changes on equilibrium states with a temperature into another, there is also a virtual version which does not change the real temperature but which acts in the virtual direction of the interpolating family of non-commutative $L^{p}$-spaces [1] to be built here on the von Neumann algebras $\mathfrak{M}:=\pi_{\beta}(\mathfrak{A})^{\prime \prime}$, defined as the GNS representation associated to a KMS state $\omega_{\beta}$. A virtual temperature $\tau$ associated with an $L^{p}$-space $L^{p}\left(\mathfrak{M} ; \omega_{\beta}\right)$ is given here as $0 \leq \tau=\beta / p \leq \beta$ (or, $T=\left(k_{B} \beta\right)^{-1} \leq p T \leq \infty$ : i.e., high temperature side) because of the restriction of $1 \leq p \leq \infty$. From the physical viewpoint, this restriction can be naturally understood as the difficulty in moving kinematically to the direction of lower temperatures caused by the possible occurrence of phase transitions which cannot be treated without the dynamical considerations.

This context of interpolation theory involves various interesting aspects, such as the extension of some key notions in the (quantum) information geometry and statistical inference theory like $\alpha$-divergences, relative entropy, Fisher information, etc., which have been traditionally treated in quantum systems with finite degrees of freedom, or even in the finite-dimensional matrix algebras $[15,13]$. On the present setting-up, it is quite natural to extend the discussions in this context to a general quantum dynamical system with infinite degrees of freedom. For instance, with $\alpha:=\frac{1}{q}-\frac{1}{p} \neq \pm 1, \frac{1}{p}+\frac{1}{q}=1$, we have an $\alpha$-divergence

$$
D^{(1 / q-1 / p)}\left(T_{1} \| T_{2}\right)=p q\left[\frac{\left\|T_{1}\right\|_{p}^{p}}{p}+\frac{\left\|T_{2}\right\|_{q}^{q}}{q}-\operatorname{Re}\left\langle T_{1}, T_{2}\right\rangle_{\phi_{0}}\right],
$$

where $\left\langle T_{1}, T_{2}\right\rangle_{\phi_{0}}$ is the pairing between $L^{p}$ and $L^{q}$ w.r.t. a faithful normal semifinite weight $\phi_{0}$ (or, a faithful normal state such as $\omega_{\beta}$ ) for $T_{1}=u_{1} \Delta_{\varphi_{1}, \phi_{0}}^{1 / p} \in$ $L^{p}\left(\mathfrak{M}, \phi_{0}\right), T_{2}=u_{2} \Delta_{\varphi_{2}, \phi_{0}}^{1 / q} \in L^{q}\left(\mathfrak{M}, \phi_{0}\right)$, with $u_{i}$ : partial isometries and $\Delta_{\varphi_{i}, \phi_{0}}$ : relative modular operator from $\phi_{0}$ to $\varphi_{i} \in \mathfrak{M}_{*,+}$. The problems related with this geometric aspect of temperature states will be discussed elsewhere. 


\section{Acknowledgement}

I would like to express my sincere thanks to Prof. Takahiro Kawai for his encouragements in my research programmes. I am very grateful to Prof. Huzihiro Araki for his remark on the relation between type III representations and the asymptotic abelianness. This work was partially supported by JSPS Grants-in-Aid (No. 15540117).

\section{References}

[1] Araki, H. and Masuda, T., Positive cones and $L^{p}$-spaces for von Neumann algebras, Publ. RIMS, Kyoto Univ., 18 (1982), 339-411; Masuda, T., $L^{p}$-spaces for von Neumann algebra with reference to a faithful normal semifinite weight, Publ. RIMS, Kyoto Univ., 19 (1983), 673-727.

[2] Bratteli, O. and Robinson, D. W., Operator Algebras and Statistical Mechanics, vol. 2, Springer-Verlag, 1981.

[3] Bros, J. and Buchholz, D., Towards a relativistic KMS condition, Nucl. Phys. B, 429 (1994), 291-318.

[4] Brunetti, R., Fredenhagen, K. and Verch, R., The generally covariant locality principle - A new paradigm for local quantum physics, Comm. Math. Phys., 237 (2003), 31-68.

[5] Buchholz, D., Ojima, I. and Roos, H., Thermodynamic properties of non-equilibrium states in quantum field theory, Ann. Phys. (N.Y.), 297 (2002), 219-242.

[6] Buchholz, D. and Verch, R., Scaling algebras and renormalization group in algebraic quantum field theory, Rev. Math. Phys., 7 (1995), 1195-1240.

[7] Dixmier, J., $C^{*}$-Algebras, North-Holland, 1977.

[8] Doplicher, S., Haag, R. and Roberts, J. E., Fields, observables and gauge transformations I \& II, Comm. Math. Phys., 13 (1969), 1-23; 15 (1969), 173-200; Local observables and particle statistics I \& II, 23 (1971), 199-230; 35 (1974), 49-85.

[9] Doplicher, S. and Roberts, J. E., Why there is a field algebra with a compact gauge group describing the superselection structure in particle physics, Comm. Math. Phys., 131 (1990), 51-107.

[10] Doplicher, S. and Roberts, J. E., Endomorphism of $\mathrm{C}^{*}$-algebras, cross products and duality for compact groups, Ann. Math., 130 (1989), 75-119; A new duality theory for compact groups, Invent. Math., 98 (1989), 157-218.

[11] Fredenhagen, K., On the modular structure of local algebras of observables, Comm. Math. Phys., 97 (1985), 79-89; Buchholz, D., D'Antoni, C. and Fredenhagen, K., The universal structure of local algebras, Comm. Math. Phys., 111 (1987), 123-135.

[12] Haag, R., Local Quantum Physics (2nd. ed.), Springer-Verlag, 1996.

[13] Hasegawa, H., $\alpha$-divergence of the non-commutative information geometry, Rep. Math. Phys., 33 (1993), 87-93; Hasegawa, H. and Petz, D., On the Riemannian metric of $\alpha$-entropies of density matrices, Lett. Math. Phys., 38 (1996), 221-225.

[14] Hugenholtz, N. M., On the factor type of equilibrium states in quantum statistical mechanics, Comm. Math. Phys., 6 (1967), 189-193; Størmer, E., Types of von Neumann algebras associated with extremal invariant states, Comm. Math. Phys., 6 (1967), 194204.

[15] Nagaoka, H. and Amari, S., Differential geometry of smooth families of probability distributions, METR, 82-7 (1982), University of Tokyo; Amari, S., Differential-Geometrical Methods in Statistics, Springer Lecture Notes in Statistics, 28 (1985).

[16] Ojima, I., Lorentz invariance vs. temperature in QFT, Lett. Math. Phys., 11 (1986), 73-80. 
[17] _ Quantum-field theoretical approach to non-equilibrium dynamics in curved space-time, pp.91-96 in Proc. 2nd Int. Symp. Foundations of Quantum Mechanics, 1986, Physical Society of Japan, 1987.

[18] Ojima, I., Non-equilibrium local states in relativistic quantum field theory, pp. 48-67 in Proc. of Japan-Italy Joint Workshop on Fundamental Problems in Quantum Physics, Sep. 2001, eds. L. Accardi and S. Tasaki, World Scientific, 2003 (available also at http://www.f.waseda.jp/stasaki/WS/Ojima.pdf).

[19] - How to formulate non-equilibrium local states in QFT?- General characterization and extension to curved spacetime-, pp. 365-384 in "A Garden of Quanta", World Scientific, 2003; e-print: cond-mat/0302283.

[20] _ A unified scheme for generalized sectors based on selection criteria -Order parameters of symmetries and of thermality and physical meanings of adjunctions-, Open Sys. Inf. Dyn., 10 (2003), 235-279; math-ph/0303009.

[21] $\ldots$, work in progress; Generalized sectors and adjunctions to control micro-macro transitions, talk presented at International Conference on Quantum Information, 2003, held at International Institute for Advanced Studies, Kyoto, Japan (November 5-7, 2003).

[22] Ozawa, M., Conditional probability and a posteriori states in quantum mechanics, Publ. RIMS, Kyoto Univ., 21 (1985), 279-295.

[23] Satake, I., Algebraic Structures of Symmetric Domains, Iwanami Shoten and Princeton University Press, 1980.

[24] Sewell, G. L., Quantum Theory of Collective Phenomena, Oxford Univ. Press, 1986.

[25] Takesaki, M., Disjointness of the KMS states of different temperatures, Comm. Math. Phys., 17 (1970), 33-41. 